\title{
Boundedness of positive operators on weighted amalgams
}

\author{
María Isabel Aguilar Cañestro and Pedro Ortega Salvador
}

* Correspondence: portega@uma. es

Análisis Matemático, Facultad de Ciencias, Universidad de Málaga, 29071 Málaga, Spain

\section{Abstract}

In this article, we characterize the pairs $(u, v)$ of positive measurable functions such that $T$ maps the weighted amalgam $\left(L^{\bar{p}}(v), \ell^{\bar{q}}\right)$ in $\left(L^{p}(u), \ell^{q}\right)$ for all $1<p, q, \bar{p}, \bar{q}<\infty$, where $T$ belongs to a class of positive operators which includes Hardy operators, maximal operators, and fractional integrals.

2000 Mathematics Subject Classification 26D10, 26D15 (42B35)

Keywords: Amalgams, Maximal operators, Weighted inequalities, Weights

\section{Introduction}

Let $u$ be a positive function of one real variable and let $p, q>1$. The amalgam $\left(L^{p}(u)\right.$, $\left.\ell^{q}\right)$ is the space of one variable real functions which are locally in $L^{p}(u)$ and globally in $\ell^{q}$. More precisely,

$$
\left(L^{p}(u), \ell^{q}\right)=\left\{f:\|f\|_{p, u, q}<\infty\right\},
$$

where

$$
\|f\|_{p, u, q}=\left\{\sum_{n \in Z}\left(\int_{n}^{n+1}|f|^{p} u\right)^{\frac{q}{p}}\right\}^{\frac{1}{q}} .
$$

These spaces were introduced by Wiener in [1]. The article [2] describes the role played by amalgams in Harmonic Analysis.

Carton-Lebrun, Heinig, and Hoffmann studied in [3] the boundedness of the Hardy operator $P f(x)=\int_{-\infty}^{x}|f|$ in weighted amalgam spaces. They characterized the pairs of weights $(u, v)$ such that the inequality

$$
\|P f\|_{p, u, q} \leq C|| f \|_{\bar{p}, v, \bar{q}}
$$

holds for all $f$, with a constant $C$ independent of $f$, whenever $1<\bar{q} \leq q<\infty$. The characterization of the pairs $(u, v)$ for (1.1) to hold in the case $1<q<\bar{q}<\infty$ has been recently completed by Ortega and Ramírez ([4]), who have also characterized the weak type inequality

$$
\|P f\|_{p, \infty ; u, q} \leq C\|f\|_{\bar{p}, v, \bar{q}^{\prime}}
$$

\section{SpringerOpen ${ }^{\circ}$}

(C) 2011 Cañestro and Salvador; licensee Springer. This is an Open Access article distributed under the terms of the Creative Commons Attribution License (http://creativecommons.org/licenses/by/2.0), which permits unrestricted use, distribution, and reproduction in any medium, provided the original work is properly cited. 


$$
\text { where }\|g\|_{p, \infty ; u, q}=\left\{\sum_{n \in \mathbb{Z}}\left\|g \mathcal{X}_{(n, n+1)}\right\|_{p, \infty, u}^{q}\right\}^{\frac{1}{q}} \text {. }
$$

There are several articles dealing with the boundedness in weighted amalgams of other operators different from Hardy's one. Specifically, Carton-Lebrun, Heinig, and Hoffmann studied in [3] weighted inequalities in amalgams for the Hardy-Littlewood maximal operator as well as for some integral operators with kernel $K(x, y)$ increasing in the second variable and decreasing in the first one. On the other hand, Rakotondratsimba ([5]) characterized some weighted inequalities in amalgams (corresponding to the cases $1<\bar{p} \leq p<\infty$ and $1<\bar{q} \leq q<\infty$ ) for the fractional maximal operators and the fractional integrals. Finally, the authors characterized in [6] the weighted inequalities for some generalized Hardy operators, including the fractional integrals of order greater than one, in all cases $1<p, \bar{p}, q, \bar{q}<\infty$, extending also results due to Heinig and Kufner [7].

Analyzing the results in the articles cited above, one can see some common features that lead to explore the possibility of giving a general theorem characterizing the boundedness in weighted amalgams of a wide family of positive operators, and providing, in such a way, a unified approach to the subject. This is the purpose of this article.

\section{The results}

We consider an operator $T$ acting on real measurable functions $f$ of one real variable and define a sequence $\left\{T_{n}\right\}_{n \in Z}$ of local operators by

$$
T_{n} f(x)=T\left(f \mathcal{X}_{(n-1, n+2)}\right)(x) \quad x \in(n-1, n+2) .
$$

We assume that there exists a discrete operator $T^{\text {d }}$, i.e., which transforms sequences of real numbers in sequences of real numbers, verifying the following conditions:

(i) There exists $C>0$ such that for all non-negative functions $f$, all $n \in \mathbb{Z}$ and all $x$ $\in(n, n+1)$, the inequality

$$
T\left(f \mathcal{X}_{(-\infty, n-1)}+f \mathcal{X}_{(n+2, \infty)}\right)(x) \leq C T^{\mathrm{d}}\left(\left\{\int_{m-1}^{m} f\right\}\right)(n)
$$

holds.

(ii) There exists $C>0$ such that for all sequences $\left\{a_{k}\right\}$ of non-negative real numbers and $n \in \mathbb{Z}$, the inequality

$$
T^{\mathrm{d}}\left(\left\{a_{k}\right\}\right)(n) \leq C T f(y),
$$

holds for all $y \in(n, n+1)$ and all non-negative $f$ such that $\int_{m-1}^{m} f=a_{m}$ for all $m$.

We also assume that $T$ verifies $T f=T|f|, T(\lambda f)=|\lambda| T f, T(f+g)(x) \leq T f(x)+T g(x)$ and $T f(x) \leq T g(x)$ if $0 \leq f(x) \leq g(x)$. 
We will say that an operator $T$ verifying all the above conditions is admissible.

There is a number of important admissible operators in Analysis. For instance: Hardy operators, Hardy-Littlewood maximal operators, Riemann-Liouville, and Weyl fractional integral operators, maximal fractional operators, etc.

Our main result is the following one:

Theorem 1. Let $1<p, q, \bar{p}, \bar{q}<\infty$. Let $u$ and $v$ be positive locally integrable functions on $\mathbb{R}$ and let $T$ be an admissible operator. Then there exists a constant $C>0$ such that the inequality

$$
\|T f\|_{p, u, q} \leq C|| f \|_{\bar{p}, v, \bar{q}} .
$$

holds for all measurable functions $f$ if and only if the following conditions hold:

(i) $T^{\mathrm{d}}$ is bounded from $\ell^{\bar{q}}\left(\left\{v_{n}\right\}\right)$ to $\ell^{q}\left(\left\{u_{n}\right\}\right)$, where $v_{n}=\left(\int_{n-1}^{n} v^{1-\bar{p}^{\prime}}\right)-\overline{\bar{q}}$ and $u_{n}=\left(\int_{n}^{n+1} u\right)^{\frac{q}{p}}$.

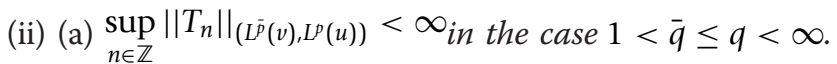

(b) $\left\{\left\|T_{n}\right\|_{\left(L^{\bar{p}}(v), L^{p}(u)\right)}\right\} \in \ell^{s}$, with $\frac{1}{s}=\frac{1}{q}-\frac{1}{\bar{q}}$, in the case $1<q<\bar{q}<\infty$.

The proof of Theorem 1 is contained in Sect. 3 .

Working as in Theorem 1, we can also prove the following weak type result:

Theorem 2. Let $1<p, q, \bar{p}, \bar{q}<\infty$. Let $u$ and $v$ be positive locally integrable functions on $\mathbb{R}$ and let $T$ be an admissible operator. Then there exists a constant $C>0$ such that the inequality

$$
\|T f\|_{p, \infty, u, q} \leq C|| f \|_{\bar{p}, v, \bar{q}}
$$

holds for all measurable functions $f$ if and only if the following conditions hold:

(i) $T^{\mathrm{d}}$ is bounded from $\ell^{\bar{q}}\left(\left\{v_{n}\right\}\right)$ to $\left.\ell^{q}\left(\left\{u_{n}\right\}\right),\right)$, with $v_{n}$ and un defined as in Theorem 1.

(ii) (a) $\sup _{n \in \mathbb{Z}}\left\|T_{n}\right\|_{\left(L^{\bar{p}}(v), L^{p, \infty}(u)\right)}<\infty_{\text {in }}$ the case $1<\bar{q} \leq q<\infty$.

(b) $\left\{\left\|T_{n}\right\|_{\left(L^{\bar{p}}(v), L^{p, \infty}(u)\right)}\right\} \in \ell^{s}$, with $\frac{1}{s}=\frac{1}{q}-\frac{1}{\bar{q}}$, in the case $1<q<\bar{q}<\infty$.

If conditions on the weights $u, v$, and $\left\{u_{n}\right\},\left\{v_{n}\right\}$ characterizing the boundedness of the operators $T_{n}$ and $T^{\mathrm{d}}$, respectively, are available in the literature, we immediately obtain, by applying Theorems 1 and 2, conditions guaranteeing the boundedness of $T$ between the weighted amalgams. In this sense, our result includes, as particular cases, most of the results cited above from the papers [3-7], as well as other corresponding to operators whose behavior on weighted amalgams has not been studied yet.

Thus, if $M^{-}$is the one-sided Hardy-Littlewood maximal operator defined by

$$
M^{-} f(x)=\sup _{h>0} \frac{1}{h} \int_{x-h}^{x}|f|,
$$


we have:

(i) The discrete operator $\left(M^{-}\right)^{\mathrm{d}}$, defined by

$$
\left(M^{-}\right)^{\mathrm{d}}\left(\left\{a_{n}\right\}\right)(j)=\sup _{k \leq j-1} \frac{1}{j-k} \sum_{i=k}^{j-1}\left|a_{i}\right|,
$$

verifies conditions (2.1) and (2.2).

(ii) The local operators $M_{n}^{-}$are defined by

$$
M_{n}^{-} f(x)=\sup _{0<h \leq x-n+1} \frac{1}{h} \int_{x-h}^{x}|f|, x \in(n-1, n+2) .
$$

(iii) If $p=\bar{p}$ and $q=\bar{q}$, there are well-known conditions on the weights $u, v$, and $\left\{u_{n}\right\}$, $\left\{v_{n}\right\}$ that characterize the boundedness of $M_{n}^{-}$and $\left(M^{-}\right)^{\mathrm{d}}$ (see, for instance [8-10]).

Therefore, we obtain the following result:

Theorem 3. The following statements are equivalent:

(i) $M^{-}$is bounded from $\left(L^{p}(w), \ell^{q}\right)$ to $\left(L^{p}(w), \ell^{q}\right)$.

(ii) $M^{-}$is bounded from $\left(L^{p}(w), \ell^{q}\right)$ to $\left(L^{p, \infty}(w), \ell^{q}\right)$.

(iii) The next conditions hold simultaneously:

$$
\begin{aligned}
& \text { (a) } w \in A_{p,(n-1, n+2)}^{-} \text {for all } n \text {, uniformly, and } \\
& \text { (b) the pair }\left(\left\{u_{n}\right\},\left\{v_{n}\right\}\right) \text { verifies the discrete Sawyer's condition } S_{q}^{-} \text {, i.e., there exists } \\
& C>0 \text { such that } \\
& \sum_{j=r}^{k}\left(\left(M^{-}\right)^{\mathrm{d}}\left(\left\{v_{n}^{1-q^{\prime}}\right\}\right)\right)^{q}(j) u_{j} \leq C \sum_{j=r}^{k} v_{j}^{1-q^{\prime}}, \\
& \text { for all } r, k \in \mathbb{Z} \text { with } r \leq k .
\end{aligned}
$$

We can state a similar result for the one-sided maximal operator $M^{+}$. In this case, the operator $\left(M^{+}\right)^{\mathrm{d}}$ defined by

$$
\left(M^{+}\right)^{\mathrm{d}}\left(\left\{a_{n}\right\}\right)(j)=\sup _{k \geq j+3} \frac{1}{k-j-2} \sum_{i=j+3}^{k}\left|a_{i}\right|,
$$

verifies conditions (2.1) and (2.2). The theorem is the next one:

Theorem 4. The following statements are equivalent:

(i) $M^{+}$is bounded from $\left(L^{p}(w), \ell^{q}\right)$ to $\left(L^{p}(w), \ell^{q}\right)$.

(ii) $M^{+}$is bounded from $\left(L^{p}(w), \ell^{q}\right)$ to $\left(L^{p, \infty}(w), \ell^{q}\right)$.

(iii) The next conditions hold simultaneously:

(a) $w \in A_{p,(n-1, n+2)}^{+}$for all $n$, uniformly, and

(b) the pair $\left(\left\{u_{n}\right\},\left\{v_{n-3}\right\}\right)$ verifies the discrete Sawyer's condition $S_{q}^{+}$, i.e., there exists $C>0$ such that

$$
\sum_{j=r}^{k}\left(\left(M^{+}\right)^{\mathrm{d}}\left(\left\{v_{n}^{1-q^{\prime}}\right\}\right)\right)^{q}(j) u_{j} \leq C \sum_{j=r}^{k} v_{j}^{1-q^{\prime}},
$$


for all $r, k \in \mathbb{Z}$ with $r \leq k$.

If $M$ is the Hardy-Littlewood maximal operator, defined by

$$
M f(x)=\sup _{x \in I} \frac{1}{|I|} \int_{I}|f|,
$$

then $M$ is admissible, with $M^{\mathrm{d}}\left(\left\{a_{n}\right\}\right)(j)=\sup _{r \leq j \leq k} \frac{1}{k-r+1} \sum_{i=r}^{k}\left|a_{i}\right|$, and there are wellknown results, due to Muckenhoupt ([11]) and Sawyer ([12]), which characterize the boundedness of $M$ in weighted Lebesgue spaces. Applying Theorems 1 and 2, we get the following result:

Theorem 5. The following statements are equivalent:

(i) $M$ is bounded from $\left(L^{p}(w), \ell^{q}\right)$ to $\left(L^{p}(w), \ell^{q}\right)$.

(ii) $M$ is bounded from $\left(L^{p}(w), \ell^{q}\right)$ to $\left(L^{p, \infty}(w), \ell^{q}\right)$.

(iii) The next conditions hold simultaneously:

(a) $w \in A_{p,(n-1, n+2)}$ for all $n$, uniformly, and

(b) the pair $\left(\left\{u_{n}\right\},\left\{v_{n}\right\}\right)$ verifies the discrete two-sided Sawyer's condition $S_{q}$, i.e., there exists $C>0$ such that

$$
\sum_{j=r}^{k}\left(M^{\mathrm{d}}\left(\left\{v_{n}^{1-q^{\prime}}\right\}\right)^{q}(j) u_{j} \leq C \sum_{j=r}^{k} v_{j}^{1-q^{\prime}}\right.
$$

for all $r, k \in \mathbb{Z}$ with $r \leq k$.

This result improves the one obtained by Carton-Lebrun, Heinig and Hofmann in [3], in the sense that the conditions we give are necessary and sufficient for the boundedness of the maximal operator in the amalgam $\left(L^{p}(w), \ell^{q}\right)$, while in [3] only sufficient conditons were given. We also prove the equivalence between the strong type inequality and the weak type inequality. The equivalence (i) $\Leftrightarrow$ (iii) in Theorem 5 is included in Rakotondratsimba's paper [5], where the proof of the admissibility of $M$ can also be found.

Finally, we will apply our results to the fractional maximal operator $M_{\alpha}, 0<\alpha<1$, defined by

$$
M_{\alpha} f(x)=\sup _{c<x<d} \frac{1}{(d-c)^{1-\alpha}} \int_{c}^{d}|f| .
$$

The proof of the admissibility of $M_{\alpha}$, with the obvious $M_{\alpha}^{\mathrm{d}}$, is implied in Rakotondratsimba's paper ([5]).

Verbitsky ([13]) in the case $1<q<p<\infty$ and Sawyer ([12]) in the case $1<p \leq q<\infty$ characterized the boundedness of $M_{\alpha}$ from $L^{p}$ to $L^{q}(w)$. These results allow us to give necessary and sufficient conditions on the weight $u$ for $M_{\alpha}$ to be bounded from $\left(L^{\bar{p}}, \ell^{\bar{q}}\right)$ to $\left(L^{p}(u), \ell^{q}\right)$. 
Before stating the theorem, we introduce the notation:

(i) If $1<\bar{q}<\infty$, we define $H: \mathbb{Z} \rightarrow \mathbb{R}$ by

$$
H(i)=\sup _{r \leq i \leq k} \frac{1}{(k-r+1)^{1-\alpha \bar{q}}} \sum_{j=r}^{k} u_{j} .
$$

(ii) If $1<\bar{q} \leq q$, we define

$$
J=\sup _{r \leq k} \frac{\left\|\mathcal{X}_{[r, k]} M_{\alpha}^{\mathrm{d}}\left(\mathcal{X}_{[r, k]}\right)\right\|_{\ell^{q}\left(\left\{u_{j}\right\}\right)} .}{(k-r+1)^{\frac{1}{\bar{q}}}}
$$

(iii) If $1<\bar{p}<\infty$ and $n \in \mathbb{Z}$, we define for $x \in(n-1, n+2)$

$$
H_{n}(x)=\sup _{x \in I \subset(n-1, n+2)} \frac{1}{|I|^{1-\alpha \bar{p}}} \int_{I} u .
$$

(iv) If $1<\bar{p}<p$ and $n \in \mathbb{Z}$, we define

$$
J_{n}=\sup _{I \subset(n-1, n+2)} \frac{\left\|\mathcal{X}_{I} M_{\alpha}\left(\mathcal{X}_{I}\right)\right\|_{L^{p}(u)}}{|I|^{\frac{1}{\bar{p}}}} .
$$

The result reads as follows.

Theorem 6. $M_{\alpha}$ is bounded from $\left(L^{\bar{p}}, \ell^{\bar{q}}\right)$ to $\left(L^{p}(u), \ell^{q}\right)$ if and only if

(i) in the case $1<\bar{p} \leq p<\infty$ and $1<\bar{q} \leq q<\infty$, $\sup _{n \in \mathbb{Z}} J_{\mathrm{n}}<\infty$ and $J<\infty$;

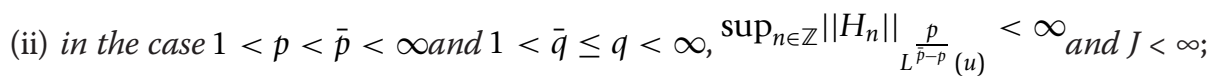

(iii) in the case $1<\bar{p} \leq p<\infty$ and $1<q<\bar{q}<\infty,\left\{J_{n}\right\}_{n} \in \ell^{s}$, where $\frac{1}{s}=\frac{1}{q}-\frac{1}{\bar{q}}$,

and $H \in \ell^{\frac{q}{\bar{q}-q}}\left(\left\{u_{j}\right\}\right)$

(iv) in the case $1<p<\bar{p}<\infty$ and $1<q<\bar{q}<\infty, \quad\left\|H_{n}\right\| \frac{p}{L^{p-p}(u)} \in \ell_{\text {and }}^{s}$

$H \in \ell^{\frac{q}{\bar{q}-q}}\left(\left\{u_{j}\right\}\right)$

\section{Proof of Theorem 1}

Let us suppose that the inequality (2.3) holds. Let $n \in \mathbb{Z}$ and let $f$ be a non-negative function supported in $(n-1, n+2)$. Then, on one hand,

$$
\left.\|f\|_{\bar{p}, v, \bar{q}}=\left\{\left(\int_{n-1}^{n} f^{\bar{p}} v\right)^{\overline{\bar{p}}}+\left(\int_{n}^{n+1} f^{\bar{p}} v\right)^{\frac{\bar{q}}{\bar{p}}}+\left(\int_{n+1}^{n+2} f^{\bar{p}} v\right)^{\frac{\bar{q}}{\bar{p}}} \leq C_{\bar{p}, \bar{q}}\right\}^{\frac{1}{\bar{q}}} \int_{n-1}^{n+2} f^{\bar{p}} v\right)^{\frac{1}{\bar{p}}}
$$


and, on the other hand,

$$
\begin{aligned}
\|T f\|_{p, u, q} & \geq\left\{\left(\int_{n-1}^{n}(T f)^{p} u\right)^{\frac{q}{p}}+\left(\int_{n}^{n+1}(T f)^{p} u\right)^{\frac{q}{p}}+\left(\int_{n+1}^{n+2}(T f)^{p} u\right)^{\frac{q}{p}}\right\}^{\frac{1}{q}} \\
& \geq C_{p, q}\left(\int_{n-1}^{n+2}(T f)^{p} u\right)^{\frac{1}{p}} \\
& \geq C_{p, q}\left(\int_{n-1}^{n+2}\left(T_{n} f\right)^{p} u\right)^{\frac{1}{p}} \\
& =C_{p, q}\left\|T_{n} f\right\|_{p, u} .
\end{aligned}
$$

Therefore, by (2.3), $T_{n}$ is bounded and $\left\|T_{n}\right\|_{\left(L^{\bar{p}}(v), L^{p}(u)\right)} \leq C$, where $C$ is a positive constant independent of $n$. Then (ii)a holds independently of the relationship between $q$ and $\bar{q}$. Let us prove that if $1<q<\bar{q}<\infty$, then (ii)b also holds.

It is well known that $\left\|T_{n}\right\|_{\left(L^{\bar{p}}(v), L^{p}(u)\right)}=\sup _{\left\{f:\|f\|_{L(p)}(v)\right.}\left\|T_{n} f\right\|_{L^{p}(u)}$. Therefore, for each $n$ there exists a non-negative measurable function $f_{n}$, with support in $(n-1, n+2)$ and with $\left\|f_{n}\right\|_{\left(L^{\bar{p}}(v),(n-1, n+2)\right)}=1$, such that $\left\|T_{n}\right\|_{\left(L^{\bar{p}}(v), L^{p}(u)\right)}<\left\|T_{n} f_{n}\right\|_{L^{p}(u)}+\frac{1}{2^{|n|}}$.

Since $\left\{\frac{1}{2^{|n|}}\right\} \in \ell^{s}$, to prove that $\left\{\left\|T_{n}\right\|_{\left(L^{\bar{p}}(v), L^{p}(u)\right)}\right\} \in \ell^{s}$ it suffices to see that $\left\{\left\|T_{n} f_{n}\right\|_{L^{p}(u)}\right\} \in \ell^{s}$.

Let $\left\{a_{n}\right\}$ be a sequence of non-negative real numbers and $f=\sum_{n} a_{n} f_{n}$. For each $n \in \mathbb{Z}$, $f(x) \geq a_{n} f_{n}(x)$ and then $T f(x) \geq a_{n} T_{n} f_{n}(x)$ for all $x \in(n-1, n+2)$. Thus,

$$
\|T f\|_{p, u, q} \geq C\left\{\sum_{n \in \mathbb{Z}}\left(\int_{n-1}^{n+2} a_{n}^{p}\left(T_{n} f_{n}\right)^{p} u\right)^{\frac{q}{p}}\right\}^{\frac{1}{q}}=C\left\{\sum_{n \in \mathbb{Z}} a_{n}^{q}\left\|T_{n} f_{n}\right\|_{L^{p}(u)}^{q}\right\}^{\frac{1}{q}} .
$$

Then, from (2.3) we deduce

$$
\begin{aligned}
\left\{\sum_{n \in \mathbb{Z}} a_{n}^{q}\left\|T_{n} f_{n}\right\|_{L^{p}(u)}^{q}\right\}^{\frac{1}{q}} & \leq C\left\{\sum_{n \in \mathbb{Z}}\left(\int_{n-1}^{n+2} f^{\bar{p}} v\right)^{\overline{\bar{p}}}\right\}^{\frac{1}{\bar{q}}} \\
& \leq C\left\{\sum_{n \in \mathbb{Z}} a_{n}^{\bar{q}}\left(\int_{n-1}^{n+2} f_{n}^{\bar{p}} v\right)^{\overline{\bar{p}}}\right\}^{\frac{1}{\bar{q}}} \\
& =C\left\{\sum_{n \in \mathbb{Z}} a_{n}^{\bar{q}}\right\} .
\end{aligned}
$$


This means that the identity operator is bounded from $\ell^{\bar{q}}$ to $\ell^{q}\left(\left\{\left\|T_{n} f_{n}\right\|_{L^{p}(u)}^{q}\right\}\right)$. Then $\left\{\left\|T_{n} f_{n}\right\|_{L^{p}(u)}\right\} \in \ell^{s}$, by applying the following lemma (see [4]).

Lemma 1. Let $1<q<\bar{q}<\infty$ and $\frac{1}{s}=\frac{1}{q}-\frac{1}{\bar{q}}$. Suppose that $\left\{u_{n}\right\}$ and $\left\{v_{n}\right\}$ are sequences of positive real numbers. The following statements are equivalent:

(i) There exists $C>0$ such that the inequality

$$
\left\{\sum_{n \in \mathbf{Z}}\left(\left|a_{n}\right| u_{n}\right)^{q}\right\}^{\frac{1}{\bar{q}}} \leq C\left\{\sum_{n \in \mathbf{Z}}\left(\left|a_{n}\right| v_{n}\right)^{\bar{q}}\right\}^{\frac{1}{\bar{q}}}
$$

holds for all sequences $\left\{a_{n}\right\}$ of real numbers.

(ii) The sequence $\left\{u_{n} v_{n}^{-1}\right\}$ belongs to the space $l^{s}$.

On the other hand, let us prove that (i) holds. If $\left\{a_{m}\right\}$ is a a sequence of non-negative real numbers and

$$
f=\sum_{m \in \mathbb{Z}} a_{m} \chi(m-1, m)\left(\int_{n-1}^{m} v^{1-\bar{p}^{\prime}}\right)^{-1} v^{1-\bar{p}^{\prime}},
$$

then $\int_{m-1}^{m} f=a_{m}, \int_{m-1}^{m} f^{\bar{p}} v=a_{m}^{\bar{p}}\left(\int_{m-1}^{m} v^{1-\bar{p}^{\prime}}\right)^{1-\bar{p}}$ and by the properties of the operator $T$ we have

$$
\begin{aligned}
\|T f\|_{p, u, q} & =\left\{\sum_{n \in \mathbb{Z}}\left(\int_{n}^{n+1}(T f)^{p}(x) u(x) \mathrm{d} x\right)^{\frac{q}{p}}\right\}^{\frac{1}{q}} \\
& \geq C\left\{\sum_{n \in \mathbb{Z}}\left(\int_{n}^{n+1} T^{\mathrm{d}}\left(\left\{\int_{m-1}^{m} f\right\}\right)^{p}(n) u(x) \mathrm{d} x\right)^{\frac{q}{p}}\right\}^{\frac{1}{q}} \\
& =C\left\{\sum_{n \in \mathbb{Z}} T^{\mathrm{d}}\left(\left\{a_{m}\right\}\right)^{q}(n)\left(\int_{n}^{n+1} u(x) \mathrm{d} x\right)^{\frac{q}{p}}\right\}^{\frac{1}{q}} \\
& \left.=\left\|T^{\mathrm{d}}\left\{a_{m}\right\}\right\|_{\ell q}\left\{u_{n}\right\}\right) .
\end{aligned}
$$


Applying (2.3) we obtain

$$
\begin{aligned}
\left\|T^{\mathrm{d}}\left\{a_{m}\right\}\right\|_{\ell q}\left(\left\{u_{n}\right\}\right) \leq C\left\{\sum_{n \in \mathbb{Z}}\left(\int_{n}^{n+1} f^{\bar{p}} v\right)^{\overline{\bar{p}}}\right\}^{\frac{1}{\bar{q}}} \\
=C\left\{\sum_{n \in \mathbb{Z}} a_{n}^{\bar{q}}\left(\int_{n-1}^{n} v^{1-\bar{p}^{\prime}}\right)^{-\frac{\bar{q}}{\bar{p}^{\prime}}}\right\}^{\frac{1}{\bar{q}}} \\
=\left\|a_{n}\right\|_{\ell \bar{q}\left(\left\{v_{n}\right\}\right)^{\prime}}
\end{aligned}
$$

which means that the discrete operator $T^{\mathrm{d}}$ is bounded from $\ell^{\bar{q}}\left(\left\{v_{n}\right\}\right)$ to $\ell^{q}\left(\left\{u_{n}\right\}\right)$, as we wished to prove.

Conversely, let us suppose that (i) and (ii) hold. Then, we have

$$
\begin{aligned}
& \|T f\|_{p, u, q} \leq C\left\{\sum_{n \in \mathbb{Z}}\left(\int_{n}^{n+1}\left(T\left(f \chi_{(-\infty, n-1)}+f \chi_{(n+2, \infty)}\right)\right)^{p} u\right)^{\frac{q}{p}}\right\}^{\frac{1}{q}} \\
& +C\left\{\sum_{n \in \mathbb{Z}}\left(\int_{n}^{n+1}\left(\operatorname{Tf} \chi_{(n-1, n+2)}\right)^{p} u\right)^{\frac{q}{p}}\right\}^{\frac{1}{q}} \\
& \leq C\left\{\sum_{n \in \mathbb{Z}}\left(T^{\mathrm{d}}\left(\left\{a_{m}\right\}\right)(n)\right)^{q}\left(\int_{n}^{n+1} u\right)^{\frac{q}{p}}\right\}^{\frac{1}{q}} \\
& +C\left\{\sum_{n \in \mathbb{Z}}\left(\int_{n}^{n+1}\left(T_{n} f\right)^{p} u\right)^{\frac{q}{p}}\right\}^{\frac{1}{q}} \\
& =C\left(I_{1}+I_{2}\right) \text {, }
\end{aligned}
$$

where $a_{m}=\int_{m-1}^{m} f$. 
Applying that $T^{\mathrm{d}}$ is bounded from $\ell^{\bar{q}}\left(\left\{v_{n}\right\}\right)$ to $\ell^{q}\left(\left\{u_{n}\right\}\right)$ and Hölder inequality, we obtain

$$
\begin{aligned}
I_{1} & \leq C\left\{\sum_{n \in \mathbb{Z}} a_{n}^{\bar{q}}\left(\int_{n-1}^{n} v^{1-\bar{p}^{\prime}}\right)^{-\overline{\bar{q}} \bar{p}^{\prime}}\right\}^{\frac{1}{\bar{q}}} \\
& =C\left\{\sum_{n \in \mathbb{Z}}\left(\int_{n-1}^{n} f\right)^{\bar{q}}\left(\int_{-1}^{n} v^{1-\bar{p}^{\prime}}\right)^{-\frac{\bar{q}}{\bar{p}^{\prime}}}\right\}^{\frac{1}{\bar{q}}} \\
& \leq C\left\{\sum_{n \in \mathbb{Z}}\left(\int_{n-1}^{n} f^{\bar{p} v}\right)^{\overline{\bar{p}}}\left(\int_{n-1}^{n} v^{1-\bar{p}^{\prime}}\right)^{\frac{\bar{q}}{\bar{p}^{\prime}}}\left(\int_{n-1}^{n} v^{1-\bar{p}^{\prime}}\right)^{-\frac{\bar{q}}{\bar{p}^{\prime}}}\right\}^{\overline{\bar{q}}} \\
& =C\left\{\sum_{n \in \mathbb{Z}}\left(\int_{n-1}^{n} f^{\bar{p} v} v\right)^{\overline{\bar{p}}}\right\}_{\overline{\bar{q}}}^{\frac{1}{\bar{q}}} \\
& =C|| f \|_{\bar{p}, v, \bar{q} .}
\end{aligned}
$$

Now we estimate $I_{2}$. If $1<\bar{q} \leq q<\infty$, since (ii)a holds, we know that the operators $T_{n}$ are uniformly bounded from $L^{p}(u,(n-1, n+2))$ to $L^{\bar{p}}(v,(n-1, n+2))$ and then

$$
\begin{aligned}
I_{2} & \leq\left\{\sum_{n \in \mathbb{Z}}\left(\int_{n-1}^{n+2}\left(T_{n} f\right)^{p} u\right)^{\frac{q}{p}}\right\}^{\frac{1}{q}} \\
& \leq C\left\{\sum_{n \in \mathbb{Z}}\left(\int_{n-1}^{n+2} f^{\bar{p}} v\right)^{\frac{q}{p}}\right\}^{\frac{1}{q}} \\
& \leq C\left\{\sum_{n \in \mathbb{Z}}\left(\int_{n-1}^{n+2} f^{\bar{p}} v\right)^{\frac{\bar{q}}{\bar{p}}}\right\}^{\frac{1}{\bar{q}}} \\
& \leq C|| f \|_{\bar{p}, v, \bar{q} .}
\end{aligned}
$$


Let us suppose, finally, that $1<q<\bar{q}<\infty$. Then (ii)b holds and, therefore,

$$
\begin{aligned}
& I_{2} \leq C\left\{\sum_{n \in \mathbb{Z}}\left(\int_{n-1}^{n+2} T_{n} f^{p} u\right)^{\frac{q}{p}}\right\}^{\frac{1}{q}} \\
& \leq C\left\{\sum_{n \in \mathbb{Z}}\left(\left\|T_{n}\right\|_{\left(L^{\bar{p}}(v), L^{p}(u)\right)}\right)^{q}\left(\int_{n-1}^{n+2} f^{\bar{p}} v\right)^{\frac{q}{\bar{p}}}\right\}^{\frac{1}{q}} \\
& \leq C\left\{\left(\sum_{n \in \mathbb{Z}}\left(\int_{n-1}^{n+2} f^{\bar{p}} v\right)^{\frac{\bar{q}}{\bar{p}}}\right)^{\frac{q}{\bar{q}}}\left(\sum_{n \in \mathbb{Z}}\left(\left\|T_{n}\right\|_{\left(L^{\bar{p}}(v), L^{p}(u)\right)}\right)^{\frac{q \bar{q}}{\bar{q}-q}}\right)^{\frac{\bar{q}-q}{\bar{q}}}\right\}^{\frac{1}{q}} \\
& =C\left\{\sum_{n \in \mathbb{Z}}\left(\int_{n-1}^{n+2} f^{\bar{p}} v\right)^{\frac{\bar{q}}{\bar{p}}}\right\}^{\frac{1}{\bar{q}}}\left(\sum_{n \in \mathbb{Z}}\left(\left\|T_{n}\right\|_{\left(L^{\bar{p}}(v), L^{p}(u)\right)}\right)^{s}\right)^{\frac{1}{s}} \\
& \leq C\|f\|_{\bar{p}, v, \bar{q}} .
\end{aligned}
$$

This finishes the proof of the theorem.

\section{Acknowledgements}

This research has been supported in part by MEC, grant MTM 2008-06621-C02-02, and Junta de Andalucía, Grants FQM354 and P06-FQM-01509.

\section{Authors' contributions}

Both authors participated similarly in the conception and proofs of the results. Both authors read and approved the final manuscript.

\section{Competing interests}

The authors declare that they have no competing interests.

Received: 8 October 2010 Accepted: 21 June 2011 Published: 21 June 2011

\section{References}

1. Wiener N: On the representation of functions by trigonometric integrals. Math Z 1926, 24:575-616.

Fournier JJF, Stewart J: Amalgams of $L^{p}$ and $\ell^{q}$. Bull Am Math Soc 1985, 13(1):1-21.

Carton-Lebrun C, Heinig HP, Hofmann SC: Integral operators on weighted amalgams. Stud Math 1994, 109(2):133-157.

4. Ortega Salvador P, Ramírez Torreblanca C: Hardy operators on weighted amalgams. Proc Roy Soc Edinburgh 2010, 140A:175-188.

5. Rakotondratsimba Y: Fractional maximal and integral operators on weighted amalgam spaces. J Korean Math Soc 1999, 36(5):855-890.

6. Aguilar Cañestro Ml, Ortega Salvador P: Boundedness of generalized Hardy operators on weighted amalgam spaces. Math Inequal Appl 2010, 13(2):305-318.

7. Heinig HP, Kufner A: Weighted Friedrichs inequalities in amalgams. Czechoslovak Math J 1993, 43(2):285-308.

8. Andersen $\mathrm{K}$ : Weighted inequalities for maximal functions associated with general measures. Trans Am Math SoC 1991, 326:907-920.

9. Martín-Reyes FJ, Ortega Salvador P, de la Torre A: Weighted inequalities for one-sided maximal functions. Trans Am Math Soc 1990, 319(2):517-534.

10. Sawyer ET: Weighted inequalities for the one-sided Hardy-Littlewood maximal functions. Trans Am Math Soc 1986, 297:53-61.

11. Muckenhoupt B: Weighted norm inequalities for the Hardy maximal function. Trans Am Math Soc 1972, 165:207-226.

12. Sawyer ET: A characterization of a two-weight norm inequality for maximal operators. Stud Math 1982, 75:1-11.

13. Verbitsky IE: Weighted norm inequalities for maximal operators and Pisier's Theorem on factorization through $L^{p, \infty}$. Integr Equ Oper Theory 1992, 15:124-153. 
doi:10.1186/1029-242X-2011-13

Cite this article as: Aguilar Cañestro and Ortega Salvador: Boundedness of positive operators on weighted amalgams. Journal of Inequalities and Applications 2011 2011:13.

Submit your manuscript to a SpringerOpen ${ }^{\odot}$ journal and benefit from:

- Convenient online submission

- Rigorous peer review

- Immediate publication on acceptance

- Open access: articles freely available online

- High visibility within the field

- Retaining the copyright to your article

Submit your next manuscript at $\gg$ springeropen.com 